\title{
DETECTION AND ATTENUATION OF FEEDBACK INDUCED HOWLING IN HEARING AIDS USING SUBBAND ZERO-CROSSING MEASURES
}

\author{
Nils Westerlund, Mattias Dahl, Nedelko Grbić \\ Blekinge Institute of Technology \\ School of Engineering \\ Department of Signal Processing
}

\begin{abstract}
A modern hearing aid should be aesthetically appealing as well as offer sufficient and adequate signal amplification. Due to the small physical size of these devices, acoustical feedback (howling) is a major problem. Apart from the annoyance and potential hearing damaging effects that howling implies, it also reduces the supplied maximum Real Ear Aided Gain (REAG). This paper proposes a novel method for subband feedback detection and cancellation, based on the zero-crossing rate measure. After splitting the hearing aid input signal into subbands, the distances between subband zero-crossings are measured. A low distance variance in a particular subband indicates that howling has arisen. The variance measure is then used to adaptively and continuously steer subband gain functions which attenuate tonal infested subbands. The method has proven to be robust and simulation indicates that it offers additional REAG of about $15 \mathrm{~dB}$.
\end{abstract}

\section{INTRODUCTION}

Acoustic feedback is a very common problem in hearing aids. This phenomenon, sometimes denoted howling or whistling, is harmful to the hearing aid user and degrades the overall performance of the device. Generally, a high REAG is desirable. However, the acoustical as well as the structural feedback of the hearing aid limit this maximum output gain. In addition, the size of modern hearing aids tend to decrease, and today inconspicuous In-TheEar (ITE) and In-The-Canal (ITC) ${ }^{1}$ hearing aids are getting more and more common. These small devices further reduce the maximum REAG since the input microphone and output loudspeaker are spatially close to each other. Hence, a tight fitting device is required to minimize the acoustic feedback. Because of this, vents are sometimes incorporated in the hearing aids, reducing the occlusion effects but - unfortunately — increasing acoustic feedback.

Another issue that must be addressed is the non-stationarity of the feedback path. It has been shown that the feedback path characteristics, regarding both frequency

\footnotetext{
${ }^{1}$ Hearing aids are sometimes classified as In-the-ear (ITE), In-TheCanal (ITC) and Behind-The-Ear (BTE).
}

response shape and phase, may vary greatly due to different acoustic environments, different hearing aid fittings and/or placement, interactions with eyeglasses etc. [1]. This call for adaptive methods for feedback reduction.

The maximum affordable REAG for different types of hearing aids has been investigated in [2]. BTE, ITE and ITC hearing aids evaluated in this study provided a mean maximum REAG of 68,59 and $51 \mathrm{~dB}$ at $2 \mathrm{kHz}$ respectively. Differences between hearing aid designs may alter these figures. Nevertheless, they may indicate at what REAG level a typical hearing aid starts to howl.

In order to increase the provided REAG in hearing aids, different methods have been employed. One approach has been to insert an adaptive notch filter in cascade with the hearing aid amplifier and adjust the notch center frequency to the largest spectral peak. Experiments have shown that this method have some limitations, e.g. tracking single harmonics in voiced speech. The added maximum stable REAG was 3-4 dB with a 12-tap adaptive filter [3]. Another method has been to change the phase relationship between the output and the input. In [1], a time varying phase shift is applied to the input signal. However, output signal distortion is increased and the method results in only a $1-2 \mathrm{~dB}$ maximum REAG increase.

Another approach is to continuously filter the hearing aid output with an estimate of the feedback path and subtract this estimated feedback signal from the hearing aid input. The methods used for feedback path estimation vary but some adaptive method such as the LeastMean-Square (LMS) algorithm [4] is common. A broadband noise is sometimes used for feedback path estimation: In [5] an internally generated pseudorandom signal was used to drive the LMS based adaptive process. With this method the additional REAG was $10-15 \mathrm{~dB}$.

In this paper, a novel method for hearing aid feedback detection and cancellation is presented. After splitting the hearing aid input signal into subbands, the distances between zero-crossings in these subbands are measured. A low distance variance in a particular subband indicates that howling has arisen. The variance measure is then used to adaptively and continuously steer subband gain functions which attenuate tonal infested subbands. A similar method was presented in [6] where the zero-crossing distance was measured using envelope tracking functions. In 


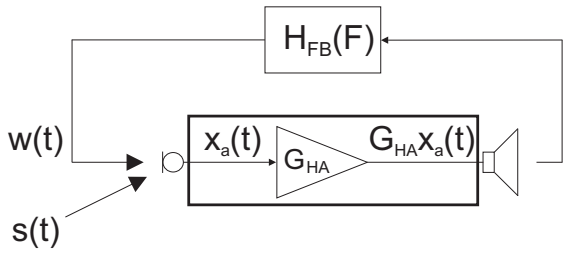

Fig. 1. A simplified hearing aid with feedback channel $H_{\mathrm{FB}}(F)$.

this paper, a more stable variance measure is used to increase algorithm reliability.

\section{SYSTEM DESCRIPTION}

A simplified illustration of a hearing aid with REAG $G_{\mathrm{HA}}$ and feedback path $H_{\mathrm{FB}}(F)$ is shown in Fig. 1. Note that $H_{\mathrm{FB}}(F)$ includes all kinds of feedback paths, e.g. vents, imperfect seal or device fitting as well as structural feedback. The signal $s(t)$ is the sound source signal and $w(t)$ is feedback signal. These two signal components add up and are amplified by the hearing aid gain before sent to the hearing aid loudspeaker. The effects of the frequency responses of the transducers are ignored in this paper.

If the criteria for unstable oscillation (howling) is fulfilled, a tonal component with exponentially increasing amplitude will arise, totally dominating the hearing aid output. The proposed method aim at detecting such tonal components using the zero-crossing measure in subbands and attenuate those subbands accordingly. This howling detection/cancellation system is placed in cascade with the user-adjustable gain $G_{\mathrm{HA}}$ (REAG). It should be mentioned that the characteristics of the hearing aid frequency response for compensating user-specific hearing curves, are not considered in this paper. An illustration of the proposed system is depicted in Fig. 2. In this figure $x(n)=$ $G_{\mathrm{HA}} x_{a}(n T)$ where $T$ is the sampling period and $n$ is the sample index.

\subsection{Zero-Crossing Rate Based Howling Detection and Attenuation}

Consider, in accordance with the previous discussion, a source sound input signal $s(t)$ entering the hearing aid via its microphone. A feedback signal component $w(t)$ is acoustically added, forming the input signal $x_{a}(t)$. The signal is amplified by the hearing aid REAG, $G_{\mathrm{HA}}$, and then properly bandlimited and sampled forming the discrete time signal $x(n)$. By filtering the input signal $x(n)$ using a bank of $K$ bandpass filters, $h_{k}(n)$, the signal is divided into $K$ subbands, each denoted by $x_{k}(n)$ where $k$ is the subband index. The subband zero-crossing measure $Z_{k}(n)$ is then calculated as

$$
Z_{k}(n)=\frac{\left|\operatorname{sgn}\left\{x_{k}(n)\right\}-\operatorname{sgn}\left\{x_{k}(n-1)\right\}\right|}{2}
$$

where the $\operatorname{sign}$ function $\operatorname{sgn}\{\cdot\}$ is defined as

$$
\operatorname{sgn}\left\{x_{k}(n)\right\}= \begin{cases}+1 & \text { if } x_{k}(n) \geqslant 0 \\ -1 & \text { if } x_{k}(n)<0\end{cases}
$$

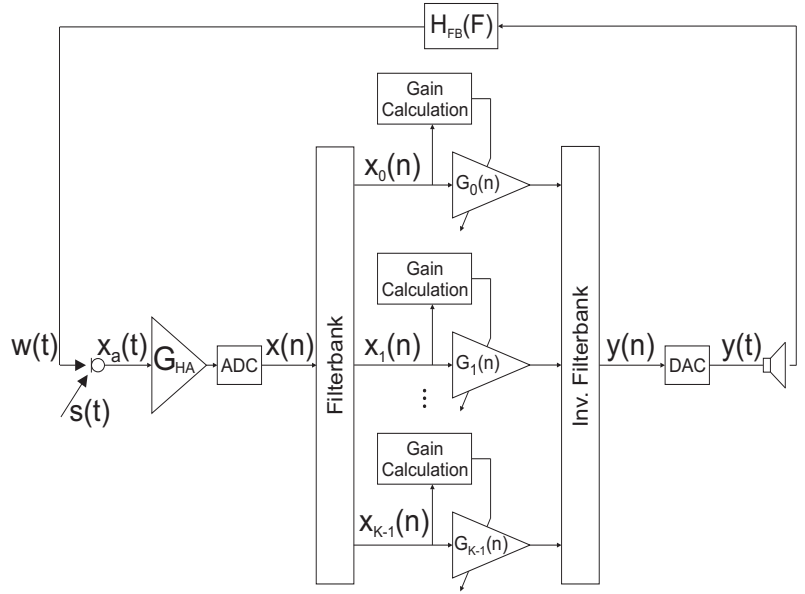

Fig. 2. The complete feedback detection/cancellation system.

Note that $Z_{k}(n)=1$ if a change in sign has occurred between sample $n$ and $n-1$ in $x_{k}(n)$ and $Z_{k}(n)=0$ otherwise. When a subband zero-crossing occurs the sample index $n$ is stored in a vector $Z_{k, i x}(l), l=\left[0 \ldots M_{k}-1\right]$, where $Z_{k, i x}(0)$ is the most recent zero-crossing sample index. The vector $Z_{k, i x}(l)$ is continuously updated in a First-In-First-Out manner (FIFO).

The distance in samples between two zero-crossings in $x_{k}(n)$ is then given by

$$
D_{k}(n)=Z_{k, i x}(0)-Z_{k, i x}\left(M_{k}-1\right)
$$

where $M_{k}$ is used to control between which zero-crossings the distance is calculated. Equation (3) results in a vector with a certain variance. A "low" variance of $D_{k}(n)$ is indicating that a tonal component is present in the subband. What is being meant by "low" is discussed in section 4.

If we treat $D_{k}(n)$ as ergodic random data an estimate of its variance $\hat{\sigma}_{D_{k}}^{2}$ over a finite length time interval of $P$ samples is now given by

$$
\hat{\sigma}_{D_{k}}^{2}=\hat{\psi}_{D_{k}}^{2}-\hat{\mu}_{D_{k}}^{2}
$$

where $\hat{\psi}_{D_{k}}^{2}$ is the estimated mean square value of $D_{k}(n)$ and $\hat{\mu}_{D_{k}}^{2}$ is the squared estimated mean value of $D_{k}(n)$, both over the sample interval $P$ [7].

The subband gain function $G_{k}(n)$ based on the estimated variance is now calculated as

$$
\begin{aligned}
& G_{k}(n)= \begin{cases}\gamma_{k} G_{k}(n-1) & \text { if } \mathcal{A} \\
\delta_{k} G_{k}(n-1) & \text { if } \mathcal{B}\end{cases} \\
& \mathcal{A}: \quad \hat{\sigma}_{D_{k}}^{2} \leqslant \alpha_{k}, \quad G_{k}(n-1)>L_{k} \\
& \mathcal{B}: \quad \hat{\sigma}_{D_{k}}^{2}>\alpha_{k}, \quad G_{k}(n-1)<1
\end{aligned}
$$

In equation (5), $\gamma_{k}$ and $\delta_{k}$ are parameters controlling the positive and negative slope of the gain function in subband $k$. Note that $\gamma_{k}<1$ and $\delta_{k}>1$. Furthermore, $\alpha_{k}$ is the variance threshold for subband $k$ : When $\hat{\sigma}_{D_{k}}^{2}$ falls below $\alpha_{k}, G_{k}(n)$ should start decreasing since this indicates that howling has occurred and that the corresponding subband 

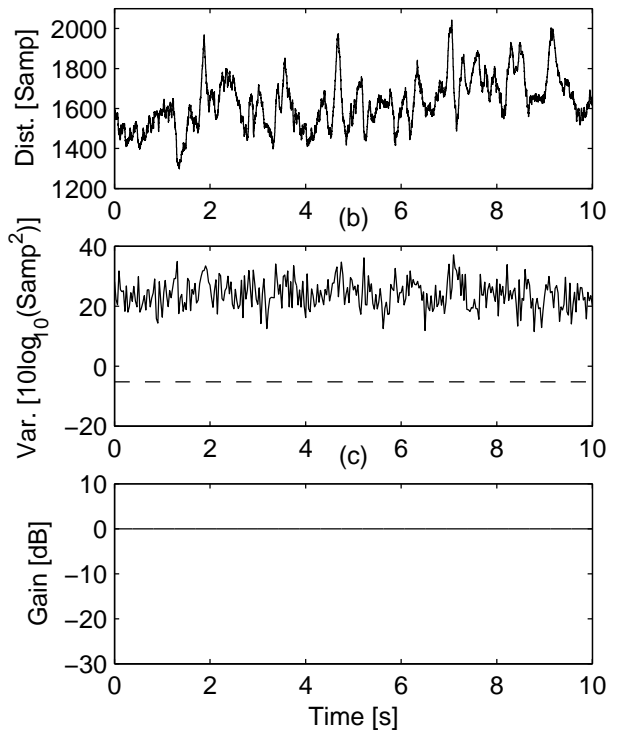

$k=4$

(d)
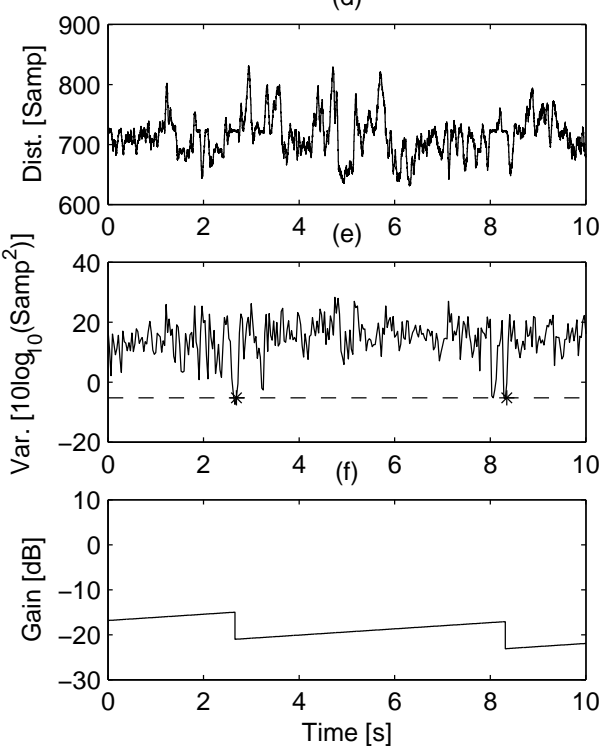

Fig. 4. Illustration of the behaviour of the zeros crossing distance measure, the variance estimate and the gain function for two different subbands. (a) Subband two, $k=2$, zero-crossing distance $D_{2}(n)$. (b) Subband two, $k=2$, estimated variance $\hat{\sigma}_{D_{2}}^{2}$. (c) Subband two, $k=2$, gain function $G_{2}(n)$. (d) Subband four, $k=4$, zero-crossing distance $D_{4}(n)$. (e) Subband four, $k=4$, estimated variance $\hat{\sigma}_{D_{4}}^{2}$. (f) Subband four, $k=4$, gain function $G_{4}(n)$. For clarity the variance measures in plots (b) and (e) have been plotted in $\mathrm{dB}$ scale and $\alpha_{k}$ is marked with a dashed line. The regions where the $\hat{\sigma}_{D_{k}}^{2} \leqslant \alpha_{k}$ are marked with asterisks.
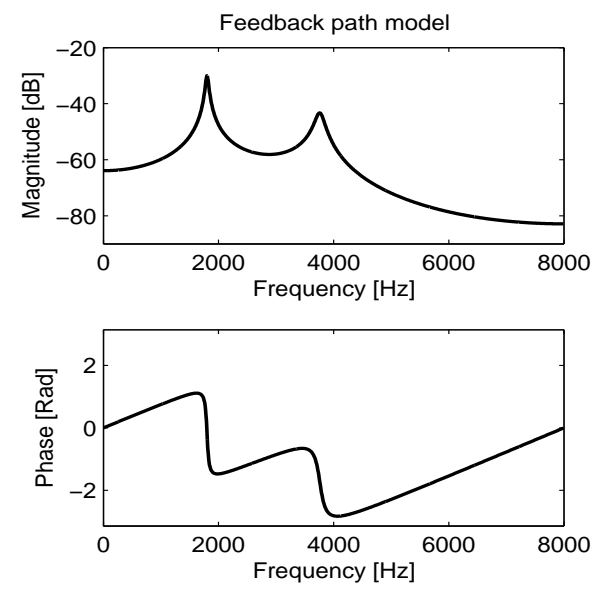

Fig. 3. Frequency and phase response of the four-pole feedback path IIR model, $\hat{H}_{F B}(f)$.

$k$ needs to be attenuated. The lower limit of $G_{k}(n)$ is denoted $L_{k}$.

Finally, the output signal $y(n)$ is obtained by applying the calculated gain to each subband signal as in

$$
y(n)=\sum_{k=0}^{K-1} G_{k}(n) x_{k}(n)
$$

\section{EVALUATION}

The input speech used for simulation was phonetically balanced sentences spoken by both males and females. The sampling frequency of the recorded sentences was $16 \mathrm{kHz}$.

For evaluation purposes a linearly spaced Finite Impulse Response (FIR) filter bank ( $\mathrm{K}=16$ subband, 256 tap filter length) with stop band attenuation of at least $50 \mathrm{~dB}$ was created using the window method.

The continuous time feedback path $H_{\mathrm{FB}}(F)$ in Fig. 1 was modelled as a four pole discrete time Infinite Impulse Response (IIR) filter $\hat{H}_{\mathrm{FB}}(f)$. The frequency and phase response of $\hat{H}_{\mathrm{FB}}(f)$ are shown in Fig. 3.

This pole setup results in a feedback path frequency response model with two local maxima approximately at the center of subband four and subband eight in the 16band filter bank. When using this model, the theoretical upper REAG limit would be approximately $30 \mathrm{~dB}$ if a flat broadband noise was used as input $s(t)$ and no feedback suppression algorithm was employed. In reality we excite the system with (non-flat spectrum) speech and hence we may increase the maximum REAG to about $35 \mathrm{~dB}$ before howling occurs.

\section{RESULTS}

To illustrate the results achieved from simulations, three of the different measures described in section 2.1 is plotted in Fig. 4 for two different subbands. In this figure plot (a), (b) and (c) shows the zero-crossing distance measure 


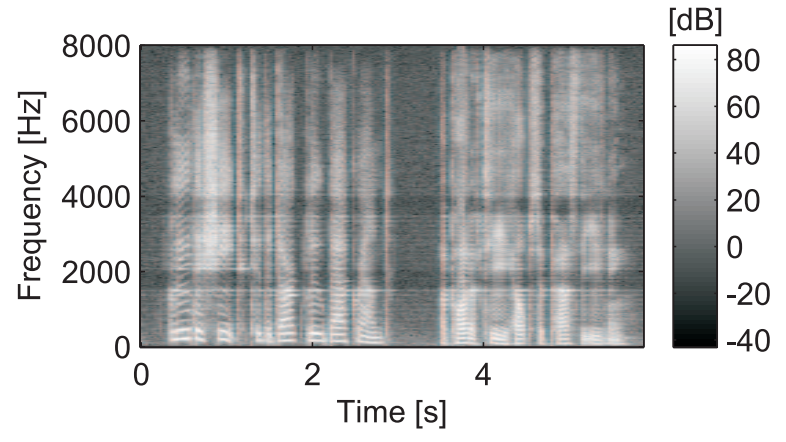

Fig. 5. Spectrogram of output.

$D_{k}(n)$, the estimated variance $\hat{\sigma}_{D_{k}}^{2}$ and the gain function $G_{k}(n)$ for subband $k=2$, respectively. The plots (d), (e) and (f) show the same measures for subband $k=4$. With the feedback path setup described in section 3 the feedback should first appear in subband four and eight. This is confirmed in the spectrogram in Fig. 5. As can be seen in this figure, the gain function is decreased in these two subbands. All other subbands remain virtually unaffected by the algorithm.

Even though a partial loss of two subbands may be audible to a hearing aid user, the overall REAG may be increased without damaging or irritating hearing aid howling. Informal listening tests indicate that an increased REAG of around $15 \mathrm{~dB}$ is possible when applying the proposed method. The parameter settings for this data run is listed in Tab. 1.

\section{CONCLUSIONS}

A subband howling detector-canceller for hearing aids was presented. A constant sample distance between subband zero-crossings indicates that feedback howling has occurred and that the subband at hand should be attenuated accordingly. The method has shown capability of increasing the provided maximum hearing aid gain by approximately $15 \mathrm{~dB}$.

\section{REFERENCES}

[1] D. K. Bustamante, T. L. Worrall, and M. J. Williamson, "Measurement and adaptive suppression of acoustic feedback in hearing aids," in Proc. Int. Conf. Acoustics, Speech and Signal Processing (ICASSP), 23-26 May 1989, pp. 2017-2020.

[2] J. Hellgren, T. Lunner, and S. Arlinger, "System identification of feedback in hearing aids," Journal of the Acoustical Society of America, vol. 105, no. 6, pp. 3481-3496, June 1999.

[3] J. A. Maxwell and P. M. Zurek, "Reducing acoustic feedback in hearing aids," IEEE Trans. On Speech and Audio Proc., vol. 3, no. 4, pp. 304-313, July 1995.

[4] B. Widrow and S. D. Stearns, Adaptive Signal Processing, Prentice-Hall, New Jersey, 1985.

[5] A. M. Engebretson, M. P. O'Connell, and F. Gong, "An adaptive feedback equalization algorithm for the cid digital hearing aid," in Proceedings of the Twelfth Annual International Conference of the IEEE Engineering in Medicine and Biology Society, 1-4 November 1990, vol. 12, pp. 2286-2287.

[6] N. Westerlund, M. Dahl, and I. Claesson, "Speech enhancement by non-stationary tonal disturbance cancellation using subband zero crossing measures," in Proc. of ISIMP04, October 2004.

[7] J. S. Bendat and A. G. Piersol, Random Data Analysis and measurement procedures, Wiley, 3rd edition, 2000.

Table 1. Parameter Selection

\begin{tabular}{l||l}
\hline Parameter & Value \\
\hline \hline$M_{k}$ & $10 K \forall k(K=16)$ \\
\hline$P$ & $500 \forall k$ \\
\hline$\left[\gamma_{k}, \delta_{k}\right]$ & {$[0.5,1.0025] \forall k$} \\
\hline$L_{k}$ & $-30 \mathrm{~dB} \forall k$ \\
\hline$\alpha_{k}$ & $0.3 \forall k$ \\
\hline
\end{tabular}

